\title{
STABILITY OF MULTIDIMENSIONAL SCALING WITH AN ERROR MODEL
}

\author{
Kanta Naito*, Sinya Murata* and Yasunori Fujikoshi*
}

\begin{abstract}
Stability of the Multidimensional Scaling (MDS) procedure is investigated under conditions where the dissimilarity data matrix involves small errors, in particular, in a somewhat ideal stochastic error model. The asymptotic performances of the solution of MDS and some related statistics are studied by using their asymptotic expansions under the model. Numerical examples which illustrate our theory are also given.
\end{abstract}

Key words and Phrases: Asymptotic expansion, Configulation, Euclidean, Multidimensional scaling, Stability.

\section{Introduction}

Multidimensional Scaling (MDS) is one of the most useful methods to represent each of $n$ objects as a point in a metric space from dissimilarity data matrix. Some properties of MDS have been studied and summarized in well-known books such as Torgerson [8], Mardia et al. ([3], Ch. 14) and Davison [2]. Let $O_{1}, \cdots, O_{n}$ be $n$ objects and let $\boldsymbol{D}=\left[d_{r s}\right]$ be the dissimilarity matrix satisfying $d_{r s}=d_{s r}>0, d_{r r}=0$. In Mardia et al. ([3], p. 395), $\boldsymbol{D}$ is called the distance matrix. Let $\boldsymbol{P}_{0}=\boldsymbol{I}_{n}-(1 / n) \mathbf{1}_{n} \mathbf{1}_{n}^{\prime}$, where $\boldsymbol{I}_{n}$ is the identity matrix and $\mathbf{1}_{n}$ is the $n$ dimensional vector whose elements are all 1 . When $\boldsymbol{B}=$ $(-1 / 2) \boldsymbol{P}_{0} \boldsymbol{D}^{(2)} \boldsymbol{P}_{0}$ is positive semidefinite (p.s.d.), $\boldsymbol{D}$ is called Euclidean. Then, it is well-known that the classical algorithm to obtain a configulation of the objects is essentially to calculate the eigenvalues and the eigenvectors of $\boldsymbol{B}$, where $\boldsymbol{D}^{(2)}=\left[d_{r s}^{2}\right]$. The matrix $\boldsymbol{B}$ is called Young-Householder (YH). transformation of $\boldsymbol{D}^{(2)}$. If $\boldsymbol{D}$ is Euclidean and the rank of $\boldsymbol{B}$ is $p$, then there exists an $n \times p$ configulation matrix $\boldsymbol{X}$ faithful to the interpoint distances given by $\boldsymbol{D}$. In this case, there exists an orthogonal matrix $\boldsymbol{H}$ and a diagonal matrix $\boldsymbol{L}=\operatorname{diag}\left\{l_{1}, \cdots, l_{p}, 0, \cdots, 0\right\}$ such that $\boldsymbol{B}=\boldsymbol{H} \boldsymbol{L} \boldsymbol{H}^{\prime}$, where $l_{1} \geq \cdots \geq l_{p}>0$ are the positive eigenvalues of $\boldsymbol{B}$ and the columns of $\boldsymbol{H}=\left[\boldsymbol{h}_{1}, \cdots, \boldsymbol{h}_{n}\right]$ are the correspondent normalized eigenvectors. Then the $n \times p$ configulation matrix is given by $\boldsymbol{X}=\boldsymbol{H}^{(p)} \boldsymbol{L}^{(p)^{12}}$, which is called a solution of MDS procedure, where $\boldsymbol{H}^{(p)}=\left[\boldsymbol{h}_{1}\right.$, $\left.\cdots, \boldsymbol{h}_{p}\right]$ and $\boldsymbol{L}^{(p)}=\operatorname{diag}\left\{l_{1}, \cdots, l_{p}\right\}$.

In this paper, we investigate the stability of the solution of the MDS procedure in the situation where the dissimilarity data matrix $\boldsymbol{D}$ involves small errors, in particular, in somewhat ideal model. The model will be realized, for example, under conditions where $\boldsymbol{D}$ is obtained as the sample mean of $m$ times observations $\boldsymbol{D}_{1}, \cdots, \boldsymbol{D}_{m}$ and that the true dissimilarity matrix $\Theta$ in our notation is Euclidean. The model is described in Section 2. In our model, the solution

Received September, 1996. Revised December, 1996. Accepted December, 1996

* Department of Mathematics, Faculty of Science, Hiroshima University, Kagamiyama 1-3-1, Higashi-Hiroshima 739, Japan. 
of MDS procedure for $\boldsymbol{D}$ is considered as an estimator of the true configulation. Stability of the MDS procedure is examined based on perturbational analysis under the model. It may be noted that the perturbational analysis of MDS has been discussed in Sibson [6]. He investigated the accuracy of the MDS method by obtaining perturbation expansion formulas of the eigenvalues and the eigenvectors of the matrix $\boldsymbol{B}$ mentioned above and the procrustes statistics which were well-discussed in Sibson [5]. However the performances of the MDS solution itself under the perturbed dissimilarity matrix were not investigated.

The purpose of this paper is to make refinements of Sibson [6]'s results by obtaining the next terms in perturbation expansions, and to extend perturbational analysis for the MDS solution itself. Further, an emphasis is put on how to make good use of these perturbation expansion formulas for inferential results with a probabilistic evaluation. Three measures of inconsistency between the estimated configulation and the true one including procrustes statistics are also considered. Asymptotic expansions of their expectations are derived. Numerical examples including real data in Davision [2] are given in Section 4. Some remarks are presented in Section 5.

\section{The stochastic model}

Some stochastic error models for MDS have been discussed by Ramsey [4]. He also discussed the maximal likelihood method in MDS. In the present paper, we assume a simple stochastic model for $\boldsymbol{D}$, given by

$$
\boldsymbol{D}=\boldsymbol{\Theta}+\varepsilon \boldsymbol{U} \quad\left(d_{r s}=\theta_{r s}+\varepsilon u_{r s}\right),
$$

where $\Theta=\left[\theta_{r s}\right]$ is the true dissimilarity matrix whose element $\theta_{r s}$ means the dissimilarity between objects $O_{r}$ and $O_{s}$, and $\theta_{r s}=\theta_{s r}>0, \theta_{r r}=0$. For the error matrix $\varepsilon \boldsymbol{U}=\left[\varepsilon u_{r s}\right], \varepsilon$ is a small quantity and the elements of $\boldsymbol{U}$ are random variables such that $u_{r r}=0, u_{r s}=u_{s r}$ and $u_{r s}$ 's for $r<s$ are independent. As usual setting, it is assumed that $E\left[u_{r s}\right]=0$ and $\operatorname{Var}\left[u_{r s}\right]=1$. This model has been considered in Sibson [6] as one of the simplest sensible error models. Then $\varepsilon$ denotes the standard deviation of each error terms. In the following, let

$$
\varepsilon=\frac{\sigma}{m^{1 / 2}}
$$

where $\sigma$ is a positive constant and $m$ is positive integer. This model is typically realized when the matrix $\boldsymbol{D}=\left[d_{r s}\right]$ is obtained by the sample mean of $m$-times independent observations. Note that in our model (2.1), it is not guaranteed that the dissimilarity data matrix $\boldsymbol{D}$ is a distance matrix. So the word Euclidean is not adopted for $D$. However, we consider the solution obtained by applying MDS procedure for $\boldsymbol{D}$ as an estimator for the solution of MDS for $\Theta$. Our main purpose is to investigate the performances of the estimator for the true solution.

Let $\boldsymbol{\Omega}$ be YH-transformation of $\Theta^{(2)}=\left[\theta_{r s}^{2}\right]$. Since the rank of $\boldsymbol{\Omega}$ is $p$ by 
assumption, there exists an orthogonal matrix $\Gamma=\left[\boldsymbol{\gamma}_{1}, \cdots, \boldsymbol{\gamma}_{n}\right]$ and a diagonal matrix $\boldsymbol{\Lambda}=\operatorname{diag}\left\{\lambda_{1}, \cdots, \lambda_{p}, 0, \cdots, 0\right\}$ such that

$$
\Omega=\Gamma \Lambda \Gamma^{\prime},
$$

where $\lambda_{1} \geq \lambda_{2} \geq \cdots \geq \lambda_{p}>0$ are the eigenvalues of $\boldsymbol{\Omega}$. We see that the true configulation in $R^{p}$ is

$$
\boldsymbol{\Xi}^{(p)}=\left[\lambda_{1}^{1 / 2} \boldsymbol{\gamma}_{1}, \cdots, \lambda_{p}^{1 / 2} \boldsymbol{\gamma}_{p}\right]=\left[\boldsymbol{\xi}_{1}, \cdots, \boldsymbol{\xi}_{p}\right] .
$$

From the model (2.1), it follows that

$$
\boldsymbol{B}=-(1 / 2) \boldsymbol{P}_{0} \boldsymbol{D}^{(2)} \boldsymbol{P}_{0}=\boldsymbol{\Omega}-\boldsymbol{\varepsilon} \boldsymbol{P}_{0} \boldsymbol{U}^{(1)} \boldsymbol{P}_{0}-(1 / 2) \varepsilon^{2} \boldsymbol{P}_{0} \boldsymbol{U}^{(2)} \boldsymbol{P}_{0}
$$

where $\boldsymbol{U}^{(1)}=\left[\theta_{r s} u_{r s}\right], \boldsymbol{U}^{(2)}=\left[u_{r s}^{2}\right]$. Thus we obtain

$$
\widetilde{B}=\Gamma^{\prime} B \Gamma=\Lambda+\varepsilon W^{(1)}+\varepsilon^{2} W^{(2)},
$$

where $\boldsymbol{W}^{(1)}=-\boldsymbol{\Gamma}^{\prime} \boldsymbol{P}_{0} \boldsymbol{U}^{(1)} \boldsymbol{P}_{0} \boldsymbol{\Gamma}$ and $\boldsymbol{W}^{(2)}=-(1 / 2) \boldsymbol{\Gamma}^{\prime} \boldsymbol{P}_{0} \boldsymbol{U}^{(2)} \boldsymbol{P}_{0} \boldsymbol{\Gamma}$. Generally, the eigenvalues of $\boldsymbol{B}$ are equal to those of $\tilde{\boldsymbol{B}}$. Further, if $\boldsymbol{h}_{\alpha}$ is the eigenvector of $\boldsymbol{B}$ belonging to the eigenvalue $\lambda_{\alpha}$, then it holds that $\boldsymbol{h}_{\alpha}=\boldsymbol{\Gamma} \tilde{\boldsymbol{h}}_{\alpha}$ for the eigenvectors $\tilde{\boldsymbol{h}}_{\alpha}$ of $\tilde{\boldsymbol{B}}$. Similarly, let the spectral decomposition of $\boldsymbol{B}$ as

$$
\boldsymbol{B}=\boldsymbol{H} \boldsymbol{L} \boldsymbol{H}^{\prime}=\boldsymbol{X} \boldsymbol{X}^{\prime},
$$

where $\boldsymbol{L}=\operatorname{diag}\left\{l_{1}, \cdots, l_{n}\right\}$ and $\boldsymbol{H}=\left[\boldsymbol{h}_{1}, \cdots, \boldsymbol{h}_{n}\right] . \quad$ In this paper we consider $\boldsymbol{H}$ and $\boldsymbol{L}$ as the estimators of $\boldsymbol{\Gamma}$ and $\boldsymbol{\Lambda}$, respectively. The estimator $\boldsymbol{x}_{\alpha}$ of $\boldsymbol{\xi}_{\alpha}$ is defined as $\boldsymbol{x}_{\alpha}=l_{\alpha}^{1 / 2} \boldsymbol{h}_{\alpha}$ whenever $l_{\alpha}>0$. Note that $\boldsymbol{\Omega}$ and $\boldsymbol{B}$ have the trivial eigenvector $n^{-1 / 2} 1_{n}$ belonging to zero eigenvalue. Thus throughout this paper, we define $\gamma_{n}$ $=n^{-1 / 2} \mathbf{1}_{n}, \boldsymbol{h}_{n}=n^{-1 / 2} \mathbf{1}_{n}$.

REMARK 1. It should be noted that the sequent discussions on our model (2.1) are regarded as an refinement of the arguments in Sibson [6]. This is recognized by observing that

$$
\boldsymbol{D}^{(2)}=\boldsymbol{\Theta}^{(2)}+2 \varepsilon \boldsymbol{U}^{(1)}+\varepsilon^{2} \boldsymbol{U}^{(2)}
$$

and hence the matrices $\boldsymbol{\Theta}^{(2)}$ and $2 \boldsymbol{U}^{(1)}$ are corresponding to the matrices $\boldsymbol{E}$ and $\boldsymbol{F}$ used in Lemma 2.2 of Sibson [6], respectively, and that in our paper the term $U^{(2)}$ in the order of $\varepsilon^{2}$ is considered but in his paper the term has been neglected.

An important measure of inconsistency between the true configulation (2.3) and its estimator

$$
\boldsymbol{X}^{(p)}=\left[\boldsymbol{x}_{1}, \cdots, x_{p}\right]
$$

in $R^{p}$ is defined as

$$
\phi(p)=\sum_{i=1}^{p}\left\|\boldsymbol{x}_{i}-\boldsymbol{\xi}_{i}\right\|^{2}=\operatorname{tr}\left(\boldsymbol{\Xi}^{(p)}-\boldsymbol{X}^{(p)}\right)^{\prime}\left(\boldsymbol{\Xi}^{(p)}-\boldsymbol{X}^{(p)}\right),
$$

which is equivalent to the sum of squared positional defferences denoted by $G\left(\boldsymbol{\Xi}^{(p)^{\prime}}, \boldsymbol{X}^{(p)^{\prime}}\right)$ in Sibson [5]. Expectation of $\phi(p)$ can be seen as the mean squared error of estimator $\boldsymbol{X}^{(p)}$. We also consider procrustes statistics 
$G_{E}\left(\boldsymbol{\Xi}^{(p)^{\prime}}, \boldsymbol{X}^{(p)^{\prime}}\right)$ and $G_{S}\left(\boldsymbol{\Xi}^{(p)^{\prime}}, \boldsymbol{X}^{(p)^{\prime}}\right)$ discussed in Sibson [6] as alternative measures and we denote these by $\phi_{E}(p)$ and $\phi_{S}(p)$, respectively. $\phi_{E}(p)$ is the minimum of $G\left(\boldsymbol{\Xi}^{(p)^{\prime}}, \boldsymbol{P} \boldsymbol{X}^{(p)^{\prime}}\right)$ for all orthogonal matrix $\boldsymbol{P}$ and it is given by

$$
\phi_{\bar{E}}(p)=\operatorname{tr} \boldsymbol{\Xi}^{(p)^{\prime}} \boldsymbol{\Xi}^{(p)}+\operatorname{tr} \boldsymbol{X}^{(p)^{\prime}} \boldsymbol{X}^{(p)}-2 \operatorname{tr}\left(\boldsymbol{\Xi}^{(p)^{\prime}} \boldsymbol{X}^{(p)} \boldsymbol{X}^{(p)^{\prime}} \boldsymbol{\Xi}^{(p)}\right)^{1 / 2} .
$$

$\phi_{s}(p)$ is the minimum of $G\left(\boldsymbol{\Xi}^{(p)^{\prime}}, u \boldsymbol{P} \boldsymbol{X}^{(p)^{\prime}}\right)$ for all orthogonal matrix $\boldsymbol{P}$ and $u>$ 0 , and it is given by

$$
\phi_{s}(p)=\operatorname{tr} \boldsymbol{\Xi}^{(p)^{\prime}} \boldsymbol{\Xi}^{(p)}-\frac{\left\{\operatorname{tr}\left(\boldsymbol{\Xi}^{(p)} \boldsymbol{X}^{(p)} \boldsymbol{X}^{(p)} \boldsymbol{\Xi}^{(p)}\right)^{1 / 2}\right\}^{2}}{\operatorname{tr} \boldsymbol{X}^{(p)^{\prime}} \boldsymbol{X}^{(p)}} .
$$

In the following section, asymptotic performances of the estimator $\boldsymbol{x}_{a}$ are investigated by considering asymptotic expansions of the mean and variance of $\boldsymbol{x}_{\alpha}$. We also derive asymptotic expansion formulas of the mean of $\phi(p), \phi_{E}(p)$ and $\phi_{S}(p)$.

\section{Stability of the MDS procedure}

In this section, we investigate the performance of the MDS procedure in model (2.1), based on asymptotic expansions of the estimators of configulations. For this, we prepare some notations. Let

$$
\boldsymbol{M}=\boldsymbol{P}_{0} \Gamma=\left[\boldsymbol{\mu}_{1} \cdots \boldsymbol{\mu}_{n}\right] .
$$

We know from definitions of $\boldsymbol{P}_{0}$ and $\boldsymbol{\gamma}_{n}$ and the orthogonality that

$$
\boldsymbol{\mu}_{\alpha}=\left(\mu_{1 \alpha}, \cdots, \mu_{n \alpha}\right)^{\prime}=\boldsymbol{\gamma}_{\alpha}
$$

for $\alpha=1, \cdots, n-1$ and $\boldsymbol{\mu}_{n}=\mathbf{0}_{n}$, the zero vector in $R^{n}$. Further let

$$
\begin{aligned}
& \boldsymbol{\mu}_{(a b)}=\left(\mu_{1 a} \mu_{1 b}, \cdots, \mu_{n a} \mu_{n b}\right)^{\prime}, \\
& \boldsymbol{\mu}_{(a b c)}=\left(\mu_{1 a} \mu_{1 b} \mu_{1 c}, \cdots, \mu_{n a} \mu_{n b} \mu_{n c}\right)^{\prime} .
\end{aligned}
$$

Our main purpose is to investigate the asymptotic performances of $\boldsymbol{x}_{\alpha}$. Throughout this section, the $\sigma^{2}$ in (2.2) is dealt with as a known constant. Further, we note that the phrase "for small $\varepsilon$ " is equivalent to "for large $m$ ". The estimator $x_{a}$ is closely related to the eigenvalues and the eigenvectors of $\boldsymbol{B}$. So at first, we give perturbation expansions of the eigenvalues and the eigenvectors of $\boldsymbol{B}$, which follow from a general result (see e.g., Siotani, Hayakawa and Fujikoshi [7]).

THEOREM 1. Assume that $\boldsymbol{D}$ has a stochastic model (2.1) and the multiplicity of the a-th eigenvalue of $\boldsymbol{\Omega}$ is one. Then

$$
\begin{aligned}
l_{\alpha}=\lambda_{\alpha}+\varepsilon w_{\alpha \alpha}^{(1)}+\varepsilon^{2}\left[w_{\alpha \alpha}^{(2)}+\sum_{\beta \neq \alpha} \lambda_{\alpha \beta}\left(w_{\alpha \beta}^{(1)}\right)^{2}\right]+O\left(\varepsilon^{3}\right), \\
\boldsymbol{h}_{\alpha}=\boldsymbol{\Gamma} \tilde{\boldsymbol{h}}_{\alpha} \\
=\boldsymbol{\gamma}_{\alpha}+\varepsilon \boldsymbol{\Gamma} \tilde{\boldsymbol{h}}_{\alpha}^{(1)}+\varepsilon^{2} \boldsymbol{\Gamma} \tilde{\boldsymbol{h}}_{\alpha}^{(2)}+O\left(\varepsilon^{3}\right),
\end{aligned}
$$

where $\boldsymbol{W}^{(1)}=\left[w_{\alpha \beta}^{(1)}\right], \boldsymbol{W}^{(2)}=\left[w_{\alpha \beta}^{(2)}\right]$ and $\lambda_{\alpha \beta}=\left(\lambda_{a}-\lambda_{\beta}\right)^{-1}$. The components of $\tilde{\boldsymbol{h}}_{a}^{(i)}=$ $\left(\tilde{h}_{a}^{(i)}, \cdots, \tilde{h}_{n \alpha}^{(i)}\right)^{\prime}(i=1,2)$ are given by 


$$
\bar{h}_{j \alpha}^{(1)}=\left\{\begin{array}{cc}
-\lambda_{j \alpha} w_{j \alpha}^{(1)}, & j \neq \alpha \\
0, & j=\alpha
\end{array}\right.
$$

and

$$
\tilde{h}_{j \alpha}^{(2)}=\left\{\begin{array}{cc}
-\lambda_{j \alpha}\left\{\lambda_{j \alpha} w_{j \alpha}^{(1)} w_{\alpha \alpha}^{(1)}+\sum_{\beta \neq \alpha} \lambda_{\alpha \beta} w_{j \beta}^{(1)} w_{\beta \alpha}^{(1)}+w_{j \alpha}^{(2)}\right\}, & j \neq \alpha \\
-(1 / 2) \sum_{\beta \neq \alpha} \lambda_{\alpha \beta}^{2}\left(w_{\alpha \beta}^{(1)}\right)^{2} & , j=\alpha .
\end{array}\right.
$$

The expansion formulas (3.1) and (3.2) up to the order of $\varepsilon$ were obtained by Sibson [6]. In fact, let $\boldsymbol{M}^{+}=\Sigma\left\{\mu_{k}^{-1} \boldsymbol{e}_{k} \boldsymbol{e}_{k}^{\prime}: \mu_{k} \neq 0\right\}$ for symmetric matrix $\boldsymbol{M}$ with spectral decomposition $\sum \mu_{k} \boldsymbol{e}_{k} \boldsymbol{e}_{k}^{\prime}$. Then we have

$$
\begin{aligned}
w_{\alpha \alpha}^{(1)} & =-\frac{1}{2} \boldsymbol{\gamma}_{\alpha}^{\prime}\left(2 \boldsymbol{U}^{(1)}\right) \boldsymbol{\gamma}_{\alpha}, \\
\boldsymbol{\Gamma} \tilde{\boldsymbol{h}}_{\alpha}^{(1)} & =\sum_{j \neq \alpha} \frac{\boldsymbol{\gamma}_{j}^{\prime} \boldsymbol{U}^{(1)} \boldsymbol{\gamma}_{\alpha}}{\lambda_{j}-\lambda_{\alpha}} \boldsymbol{\gamma}_{j} \\
& =-\left(\boldsymbol{\Omega}-\lambda_{\alpha} \boldsymbol{I}\right)^{+}\left[-\frac{1}{2} \boldsymbol{P}_{0}\left(2 \boldsymbol{U}^{(1)}\right) \boldsymbol{P}_{0}\right] \boldsymbol{\gamma}_{\alpha},
\end{aligned}
$$

which are equal to the results given in Lemma 2.2 of Sibson [6]. By using Theorem 1, we get the following theorems.

THEOREM 2. Under the conditions of Theorem 1, the mean and the variance of $l_{\alpha}$ can be expanded for small $\varepsilon$ as

$$
\begin{aligned}
& E\left[l_{\alpha}\right]=\lambda_{\alpha}+\varepsilon^{2}\left\{\frac{\boldsymbol{\mu}_{\alpha}^{\prime} \boldsymbol{\mu}_{\alpha}}{2}+\sum_{\beta \neq \alpha} \lambda_{\alpha \beta}\left\{\boldsymbol{\mu}_{(\alpha \alpha)}^{\prime} \Theta^{(2)} \boldsymbol{\mu}_{(\beta \beta)}+\boldsymbol{\mu}_{(\alpha \beta)}^{\prime} \Theta^{(2)} \boldsymbol{\mu}_{(\alpha \beta)}\right\}\right\} \\
& +O\left(\varepsilon^{3}\right) \text {, } \\
& \text { (3.6) } \operatorname{Var}\left[l_{\alpha}\right]=2 \varepsilon^{2} \boldsymbol{\mu}_{(\alpha \alpha)}^{\prime} \Theta^{(2)} \boldsymbol{\mu}_{(\alpha \alpha)}+O\left(\varepsilon^{3}\right) \text {. }
\end{aligned}
$$

THEOREM 3. Under the conditions of Theorem 1 , the mean of $\boldsymbol{h}_{\alpha}=\left(h_{1 \alpha}\right.$, $\left.\cdots, h_{n \alpha}\right)^{\prime}$ and the variance of $h_{j \alpha}$ can be expanded for small $\varepsilon$ as

$$
\begin{aligned}
E\left[\boldsymbol{h}_{\alpha}\right]=\boldsymbol{\gamma}_{\alpha} & +\varepsilon^{2} \boldsymbol{\Gamma} \boldsymbol{\eta}_{\alpha}+O\left(\varepsilon^{3}\right), \\
\operatorname{Var}\left[h_{j \alpha}\right]= & \varepsilon^{2} \sum_{l \neq \alpha} \sum_{k \neq \alpha} \gamma_{j l} \gamma_{j k} \lambda_{l \alpha} \lambda_{k \alpha}\left\{\boldsymbol{\mu}_{(l \alpha)}^{\prime} \Theta^{(2)} \boldsymbol{\mu}_{(k \alpha)}+\boldsymbol{\mu}_{(l k)}^{\prime} \Theta^{(2)} \boldsymbol{\mu}_{(\alpha \alpha)}\right\} \\
& +O\left(\varepsilon^{3}\right)
\end{aligned}
$$

respectively, where $\eta_{\alpha}=\left(\eta_{1 \alpha}, \cdots, \eta_{n \alpha}\right)^{\prime}$ with the components

$$
\eta_{j \alpha}=-\lambda_{j \alpha}\left\{2 \lambda_{j \alpha} \boldsymbol{\mu}_{(j \alpha)}^{\prime} \Theta^{(2)} \boldsymbol{\mu}_{(\alpha \alpha)}+\frac{1}{2} \boldsymbol{\mu}_{j}^{\prime} \boldsymbol{\mu}_{\alpha}+\sum_{\beta \neq \alpha} \lambda_{\alpha \beta}\left\{\boldsymbol{\mu}_{(j \alpha)}^{\prime} \boldsymbol{\Theta}^{(2)} \boldsymbol{\mu}_{(\beta \beta)}+\boldsymbol{\mu}_{(j \beta)}^{\prime} \boldsymbol{\Theta}^{(2)} \boldsymbol{\mu}_{(\alpha \alpha)}\right\}\right\}
$$

for $j \neq \alpha$,

$$
\eta_{\alpha \alpha}=-\frac{1}{2} \sum_{\beta \neq \alpha} \lambda_{\alpha \beta}^{2}\left\{\boldsymbol{\mu}_{(\alpha \beta)}^{\prime} \Theta^{(2)} \boldsymbol{\mu}_{(\alpha \beta)}+\boldsymbol{\mu}_{(\alpha \alpha)}^{\prime} \Theta^{(2)} \boldsymbol{\mu}_{(\beta \beta)}\right\}
$$

We note that in our model the biases of $l_{\alpha}$ and $\boldsymbol{h}_{\alpha}$ appear in the term of $O\left(\varepsilon^{2}\right)$. Sibson mentioned that the error in the squared distances are biased; if there is any unbiasedness, it is likely to be in errors in the distances rather than 
in the squared distances. We consider certainly the model (2.1) with error in the distances. The model (2.1) induces the perturbed squared distance matrix (2.4). We see from Theorem 1 that the term of $O(\varepsilon)$ of $l_{\alpha}$ and $\boldsymbol{h}_{\alpha}$ are $w_{a \alpha}^{(1)}$ and $\boldsymbol{\Gamma} \tilde{\boldsymbol{h}}_{a}^{(1)}$, respectively. From (3.3) and (3.4), we observe that both of $\boldsymbol{w}_{a \alpha}^{(1)}$ and $\boldsymbol{\Gamma} \tilde{\boldsymbol{h}}_{a}^{(1)}$ are linear combinations of $\boldsymbol{u}_{\tau s}$, so that the bias does not appear in the term of $O(\varepsilon)$. However if $E[\boldsymbol{U}] \neq \boldsymbol{O}$, the bias appears in the terms of $O(\varepsilon)$. For example, the bias of $l_{a}$ is given by

$$
E\left[w_{a a}^{(1)}\right]=E\left[-\frac{1}{2} \boldsymbol{\gamma}_{\alpha}^{\prime}\left(2 \boldsymbol{U}^{(1)}\right) \boldsymbol{\gamma}_{a}\right]
$$

as in Proposition 2.3 in Sibson [6].

Let

$$
\begin{aligned}
& l_{\alpha}^{(1)}=w_{\alpha \alpha}^{(1)}, \\
& l_{\alpha}^{(2)}=w_{a \alpha}^{(2)}+\sum_{\beta \neq \alpha} \lambda_{\alpha \beta}\left(w_{a \beta}^{(1)}\right)^{2}
\end{aligned}
$$

and

$$
\boldsymbol{\Gamma} \tilde{\boldsymbol{h}}_{\alpha}^{(i)}=\boldsymbol{h}_{\alpha}^{(i)}=\left(h_{\alpha}^{(i)}, \cdots, h_{n \alpha}^{(i)}\right)^{\prime}
$$

for $i=1,2$. Then the equation (3.1) and (3.2) are expressed as

$$
\begin{aligned}
& l_{\alpha}=\lambda_{\alpha}+\varepsilon l_{\alpha}^{(1)}+\varepsilon^{2} l_{\alpha}^{(2)}+O\left(\varepsilon^{3}\right), \\
& \boldsymbol{h}_{a}=\boldsymbol{\gamma}_{\alpha}+\varepsilon \boldsymbol{h}_{\alpha}^{(1)}+\varepsilon^{2} \boldsymbol{h}_{\alpha}^{(2)}+O\left(\varepsilon^{3}\right),
\end{aligned}
$$

respectively. From definition $\boldsymbol{x}_{\alpha}=l_{\alpha}^{1 / 2} \boldsymbol{h}_{\alpha}, \boldsymbol{x}_{a}$ can be expanded as follow;

$$
x_{\alpha}=\xi_{\alpha}+\varepsilon x_{a}^{(1)}+\varepsilon^{2} x_{\alpha}^{(2)}+\cdots,
$$

where

$$
x_{a}^{(1)}=\left(x_{1 \alpha}^{(1)}, \cdots, x_{n a}^{(1)}\right)^{\prime}=\lambda_{\alpha}^{1 / 2} h_{\alpha}^{(1)}+\frac{l_{a}^{(1)}}{2 \lambda_{a}^{1 / 2}} \gamma_{a}
$$

and

$$
\begin{aligned}
x_{\alpha}^{(2)} & =\left(x_{1 \alpha}^{(2)}, \cdots, x_{n \alpha}^{(2)}\right)^{\prime} \\
& =\frac{1}{8 \lambda_{\alpha}}\left\{4 l_{\alpha}^{(2)} \lambda_{\alpha}^{1 / 2}-\frac{l_{a}^{(1) 2}}{\lambda_{\alpha}^{1 / 2}}\right\}+\frac{l_{\alpha}^{(1)}}{2 \lambda_{\alpha}^{1 / 2}} \boldsymbol{h}_{\alpha}^{(1)}+\lambda_{a}^{1 / 2} \boldsymbol{h}_{a}^{(2)} .
\end{aligned}
$$

Therefore, we now obtain the asymptotic expansion formulas for the mean and the variance of the estimator $x_{\alpha}$ as follows.

THEOREM 4. Under the conditions of Theorem 1, the mean of $\boldsymbol{x}_{\alpha}=\left(x_{1 \alpha}\right.$, $\left.\cdots, x_{n a}\right)^{\prime}$ and the variance of $x_{j \alpha}$ can be expanded for small $\varepsilon$ as

(3.8) $E\left[\boldsymbol{x}_{\alpha}\right]=\boldsymbol{\xi}_{\alpha}$

$$
\begin{aligned}
& +\varepsilon^{2}\left[\left\{\frac{1}{2 \lambda_{\alpha}^{1 / 2}}\left(\frac{\boldsymbol{\mu}_{\alpha}^{\prime} \boldsymbol{\mu}_{\alpha}}{2}+\sum_{\beta \neq \alpha} \lambda_{\alpha \beta}\left\{\boldsymbol{\mu}_{(\alpha \alpha)}^{\prime} \Theta^{(2)} \boldsymbol{\mu}_{(\beta \beta)}+\boldsymbol{\mu}_{(\alpha \beta)}^{\prime} \boldsymbol{\Theta}^{(2)} \boldsymbol{\mu}_{(\alpha \beta)}\right\}\right)\right.\right. \\
& \left.\left.-\frac{\boldsymbol{\mu}_{(\alpha \alpha)}^{\prime} \boldsymbol{\Theta}^{(2)} \boldsymbol{\mu}_{(\alpha a)}}{4 \lambda_{\alpha}^{3 / 2}}\right\} \boldsymbol{\gamma}_{\alpha}+\frac{1}{2 \lambda_{\alpha}^{1 / 2}} \Gamma \zeta_{\alpha}+\lambda_{\alpha}^{1 / 2} \Gamma \boldsymbol{\eta}_{\alpha}\right]+O\left(\varepsilon^{3}\right),
\end{aligned}
$$




$$
\begin{aligned}
\operatorname{Var}\left[x_{j \alpha}\right]= & \varepsilon^{2}\left[\lambda_{\alpha} \sum_{l \neq \alpha} \sum_{k \neq a} \gamma_{j i} \gamma_{j k} \lambda_{l a} \lambda_{k \alpha}\left\{\boldsymbol{\mu}_{(l \alpha)}^{\prime} \Theta^{(2)} \boldsymbol{\mu}_{(k a)}+\boldsymbol{\mu}_{(l k)}^{\prime} \Theta^{(2)} \boldsymbol{\mu}_{(a \alpha)}\right\}\right. \\
& \left.+\frac{\gamma_{j a}^{2}}{2 \lambda_{\alpha}} \boldsymbol{\mu}_{(\alpha \alpha)}^{\prime} \Theta^{(2)} \boldsymbol{\mu}_{(\alpha \alpha)}+\gamma_{j a} \sum_{k=1}^{n} \gamma_{j k} \zeta_{k \alpha}\right]+O\left(\varepsilon^{3}\right),
\end{aligned}
$$

where $\zeta_{\alpha}=\left(\zeta_{1 \alpha}, \cdots, \zeta_{n \alpha}\right)^{\prime}$ with the entries

$$
\zeta_{j \alpha}=\left\{\begin{array}{cc}
-2 \lambda_{j \alpha} \mu_{(j \alpha)}^{\prime} \Theta^{(2)} \mu_{(\alpha \alpha)}, & j \neq \alpha \\
0, & j=\alpha .
\end{array}\right.
$$

The number $m$ in model (2.1) can be regarded as the number of replications or a measure of confidency of the dissimilarity matrix. It should be noted from (3.8) that if $\varepsilon$ is sufficiently small ( $m$ is sufficiently large), then the effect of the second term in (3.8) seems to be small.

Next, we give some asymptotic results for the distribution of $\boldsymbol{x}_{\boldsymbol{\alpha}}$ for small $\varepsilon$ (large $m$ ). In what follows, we assume that $u_{r s}$ for $r<s$ are i.i.d. with $N(0$, 1), the standard normal distribution. Our derivation is based on a perturbation method whose validity have been discussed in several authors (see, e.g., Bhattacharya and Ghosh [1]). First, it is easy to see that under conditions of theorem 1, $\left\{\boldsymbol{x}_{\alpha}-\boldsymbol{\xi}_{\alpha}\right\} / \varepsilon$ is asymptotically distributed as $N_{n}\left(\mathbf{0}_{n}, \boldsymbol{\Sigma}\right), n$ variate normal distribution with mean vector $\boldsymbol{0}_{n}$ and covariance matrix $\Sigma$, where $\Sigma=$ $\left[\sigma_{i j}\right]$ with the components

$$
\begin{aligned}
\sigma_{i j}= & \lambda_{\alpha} \sum_{r \neq \alpha} \sum_{s \neq \alpha} \gamma_{i r} \gamma_{j s} \lambda_{r \alpha} \lambda_{s \alpha}\left\{\boldsymbol{\mu}_{(r s)}^{\prime} \Theta^{(2)} \boldsymbol{\mu}_{(\alpha \alpha)}+\boldsymbol{\mu}_{(r \alpha)}^{\prime} \Theta^{(2)} \boldsymbol{\mu}_{(s \alpha)}\right\} \\
& +\sum_{\beta \neq \alpha}\left\{\gamma_{i \beta} \gamma_{j \alpha}+\gamma_{i \alpha} \gamma_{j \beta}\right\} \lambda_{\alpha \beta} \boldsymbol{\mu}_{(\alpha \alpha)}^{\prime} \Theta^{(2)} \boldsymbol{\mu}_{(\alpha \beta)} \\
& +\frac{1}{2 \lambda_{\alpha}} \gamma_{i \alpha} \gamma_{j a} \boldsymbol{\mu}_{(\alpha \alpha)}^{\prime} \Theta^{(2)} \boldsymbol{\mu}_{(a \alpha)}
\end{aligned}
$$

for $i \neq \alpha$ and $j \neq \alpha$,

$$
\sigma_{i \alpha}=\sigma_{\alpha i}=\frac{\gamma_{i \alpha} \gamma_{\alpha \alpha}}{2 \lambda_{a}} \boldsymbol{\mu}_{(\alpha \alpha)}^{\prime} \Theta^{(2)} \boldsymbol{\mu}_{(\alpha \alpha)}+\gamma_{\alpha a} \sum_{\beta \neq \alpha} \gamma_{i \beta} \lambda_{\beta \alpha} \mu_{(\beta a)}^{\prime} \Theta^{(2)} \boldsymbol{\mu}_{(\alpha a)},
$$

for $i \neq \alpha$ and

$$
\sigma_{\alpha \alpha}=\frac{\gamma_{\alpha a}^{2}}{2 \lambda_{\alpha}} \boldsymbol{\mu}_{(\alpha \alpha)}^{\prime} \Theta^{(2)} \boldsymbol{\mu}_{(\alpha \alpha)}
$$

From the equation (3.7), we see that $x_{j a}^{(1)}$ is written as

$$
x_{j \alpha}^{(1)}=\sum_{r<s} x_{j \alpha}^{(1)}(r, s) u_{r s},
$$

where

$$
\begin{aligned}
x_{j a}^{(1)}(r, s) & =\lambda_{a}^{1 / 2} \sum_{k \neq \alpha} \gamma_{j k} \lambda_{k \alpha}\left(\mu_{r k} \mu_{s \alpha}+\mu_{s k} \mu_{r a}\right) \theta_{r s}+\frac{\gamma_{j \alpha}}{2 \lambda_{\alpha}^{1 / 2}}\left\{-2 \mu_{r a} \mu_{s a} \theta_{r s}\right\} \\
& \equiv \lambda_{a}^{1 / 2} h_{j a}^{(1)}(r, s)+\frac{\gamma_{j \alpha}}{2 \lambda_{a}^{1 / 2}} l_{a}^{(\mathrm{I})}(r, s),
\end{aligned}
$$




$$
\begin{aligned}
& h_{j a}^{(1)}(r, s)=\sum_{k \neq a} \gamma_{j k} \lambda_{k a}\left(\mu_{r k} \mu_{s \alpha}+\mu_{s k} \mu_{r \alpha}\right) \theta_{r s}, \\
& l_{\alpha}^{(1)}(r, s)=-2 \mu_{r a} \mu_{s a} \theta_{r s} .
\end{aligned}
$$

Further, letting

$$
\begin{aligned}
l_{\alpha}^{(2)}(r, s ; p, q)= & -\mu_{r \alpha} \mu_{s a} \delta_{p q}^{r s} \\
& +\sum_{\beta \neq \alpha} \lambda_{\alpha \beta}\left(\mu_{r \alpha} \mu_{s \beta}+\mu_{s \alpha} \mu_{r \beta}\right)\left(\mu_{p \alpha} \mu_{q \beta}+\mu_{q \alpha} \mu_{p \beta}\right) \theta_{r s} \theta_{p q},
\end{aligned}
$$

it holds that

$$
l_{a}^{(2)}=\sum_{r<s} \sum_{p<q} l_{a}^{(2)}(r, s ; p, q) u_{r s} \mu_{p q}
$$

where

$$
\delta_{p q}^{r s}=\left\{\begin{array}{l}
1, r=p \text { and } s=q \\
0, \text { otherwise }
\end{array}\right.
$$

Similarly, let

$$
\begin{aligned}
h_{j a}^{(2)}(r, s ; p, q)= & \sum_{c \neq \alpha} \gamma_{j c} \lambda_{c a}\left(\mu_{r c} \mu_{s a}+\mu_{s c} \mu_{r a}\right)\left\{\frac{1}{2} \delta_{p q}^{r s}-2 \lambda_{c \alpha} \mu_{p \alpha} \mu_{q \alpha} \theta_{r s} \theta_{p q}\right\} \\
& -\sum_{\beta \neq a}\left(\mu_{p a} \mu_{q \beta}+\mu_{q \alpha} \mu_{p \beta}\right)\left\{\lambda_{\alpha \beta} \sum_{c \neq a} \gamma_{j c} \lambda_{c a}\left(\mu_{r c} \mu_{s \beta}+\mu_{s c} \mu_{r \beta}\right)\right. \\
& \left.+\frac{1}{2} \gamma_{j a} \lambda_{\alpha \beta}^{2}\left(\mu_{r a} \mu_{s \beta}+\mu_{s a} \mu_{r \beta}\right)\right\} \theta_{r s} \theta_{p q},
\end{aligned}
$$

then

$$
\begin{aligned}
& h_{j a}^{(1)}=\sum_{r<s} h_{j a}^{(1)}(r, s) u_{r s}, \\
& h_{j a}^{(2)}=\sum_{r<s} \sum_{p<q} h_{j a}^{(2)}(r, s ; p, q) u_{r s} u_{p q} .
\end{aligned}
$$

By using these expressions, we obtain that

$$
x_{j a}^{(2)}=\sum_{r<s} \sum_{p<q} x_{j a}^{(2)}(r, s ; p, q) u_{r s} u_{p q}
$$

where

$$
\begin{aligned}
x_{j \alpha}^{(2)}(r, s ; p, q)= & \frac{\gamma_{j \alpha}}{2 \lambda_{\alpha}^{1 / 2}} l_{a}^{(2)}(r, s ; p, q)-\frac{\gamma_{j \alpha}}{8 \lambda_{a}^{3 / 2}} l_{\alpha}^{(1)}(r, s) l_{\alpha}^{(1)}(p, q) \\
& +\frac{1}{2 \lambda_{a}^{1 / 2}} l_{\alpha}^{(1)}(p, q) h_{j \alpha}^{(1)}(r, s)+\lambda_{\alpha}^{1 / 2} h_{j \alpha}^{(2)}(r, s ; p, q) .
\end{aligned}
$$

A simple but somewhat lengthy computation yields an asymptotic expansion of the distribution of $x_{j a}$ as the following theorem.

THEOREM 5. Under the conditions of theorem 1 with $u_{r s} \sim N(0,1)$, the distribution of 


$$
z_{j \alpha}=\left\{x_{j \alpha}-\xi_{j \alpha}\right\} /\left(\varepsilon q_{2}\right)
$$

can be expanded for small $\varepsilon$ as

$$
P\left\{z_{j \alpha} \leq y\right\}=\Phi(y)-\varepsilon \varphi(y)\left\{\frac{q_{1}}{q_{2}}+\frac{q_{3}}{q_{2}^{3}}\left(y^{2}-1\right)\right\}+O\left(\varepsilon^{2}\right),
$$

where $\Phi(y)$ and $\varphi(y)$ are the cumulative distribution and the density functions of $N(0,1)$, respectively, and

$$
\begin{aligned}
q_{1}=E\left[x_{j \alpha}^{(2)}\right]= & \lambda_{\alpha}^{1 / 2} q_{1}^{(1)}+\frac{1}{2 \lambda_{\alpha}^{1 / 2}} q_{1}^{(2)}+\frac{\gamma_{j \alpha}}{2 \lambda_{a}^{1 / 2}} q_{1}^{(3)}-\frac{\gamma_{j \alpha}}{8 \lambda_{\alpha}^{3 / 2}} q_{1}^{(4)} \\
q_{2}^{2}=\sigma_{j j}^{2}= & \lambda_{\alpha} \sum_{\beta \neq \alpha} \sum_{\nu \neq \alpha} \lambda_{\beta \alpha} \lambda_{\nu \alpha \alpha} \gamma_{j \beta} \gamma_{j \nu}\left\{\boldsymbol{\mu}_{(\alpha \alpha)}^{\prime} \Theta^{(2)} \boldsymbol{\mu}_{(\beta \nu)}+\boldsymbol{\mu}_{(\alpha \beta)}^{\prime} \Theta^{(2)} \boldsymbol{\mu}_{(\alpha \nu)}\right\} \\
& +\frac{\gamma_{j \alpha}^{2}}{2 \lambda_{\alpha}} \boldsymbol{\mu}_{(\alpha \alpha)}^{\prime} \Theta^{(2)} \boldsymbol{\mu}_{(\alpha \alpha)}+2 \gamma_{j \alpha} \sum_{\beta \neq \alpha} \gamma_{j \beta} \lambda_{\alpha \beta} \boldsymbol{\mu}_{(\alpha \alpha)}^{\prime} \Theta^{(2)} \boldsymbol{\mu}_{(\alpha \beta)} .
\end{aligned}
$$

The coefficients $q_{1}^{(k)}(k=1, \cdots, 4)$ are given as

$$
\begin{aligned}
q_{1}^{(1)}= & E\left[h_{j \alpha}^{(2)}\right] \\
= & -\frac{\gamma_{j \alpha}}{2} \sum_{\beta \neq \alpha} \lambda_{\alpha \beta}^{2}\left\{\boldsymbol{\mu}_{(\alpha \alpha)}^{\prime} \Theta^{(2)} \boldsymbol{\mu}_{(\beta \beta)}+\boldsymbol{\mu}_{(\alpha \beta)}^{\prime} \Theta^{(2)} \boldsymbol{\mu}_{(\alpha \beta)}\right\} \\
& -\sum_{\beta \neq \alpha} \gamma_{j \beta} \lambda_{\beta \alpha}\left\{2 \lambda_{\beta \alpha} \boldsymbol{\mu}_{(\alpha \alpha)}^{\prime} \Theta^{(2)} \boldsymbol{\mu}_{(\alpha \beta)}+\frac{1}{2} \boldsymbol{\mu}_{a}^{\prime} \boldsymbol{\mu}_{\beta}\right. \\
& \left.+\sum_{\nu \neq \alpha} \lambda_{\alpha \nu}\left\{\boldsymbol{\mu}_{(\alpha \nu)}^{\prime} \Theta^{(2)} \boldsymbol{\mu}_{(\beta \nu)}+\boldsymbol{\mu}_{(\alpha \beta)}^{\prime} \Theta^{(2)} \boldsymbol{\mu}_{(\nu \nu)}\right\}\right\}, \\
q_{1}^{(2)}= & E\left[l_{\alpha}^{(1)} h_{j \alpha}^{(1)}\right] \\
= & -2 \sum_{\beta \neq \alpha} \gamma_{j \beta} \lambda_{\beta \alpha} \boldsymbol{\mu}_{(\alpha \alpha)}^{\prime} \Theta^{(2)} \boldsymbol{\mu}_{(\alpha \beta)}, \\
q_{1}^{(3)}= & E\left[l_{\alpha}^{(2)}\right] \\
= & \frac{\boldsymbol{\mu}_{\alpha}^{\prime} \boldsymbol{\mu}_{\alpha}}{2}+\sum_{\beta \neq \alpha} \lambda_{\alpha \beta}\left\{\boldsymbol{\mu}_{(\alpha \alpha)}^{\prime} \Theta^{(2)} \boldsymbol{\mu}_{(\beta \beta)}+\boldsymbol{\mu}_{(\alpha \beta)}^{\prime} \Theta^{(2)} \boldsymbol{\mu}_{(\alpha \beta)}\right\}, \\
q_{1}^{(4)}= & E\left[\left\{l_{\alpha}^{(1)}\right\}^{2}\right] \\
= & 2 \boldsymbol{\mu}_{(\alpha \alpha)}^{\prime} \Theta^{(2)} \boldsymbol{\mu}_{(\alpha \alpha)},
\end{aligned}
$$

and $q_{3}$ is the quantity obtained by substituting $x_{j a}^{(1)}(r, s)$ and $x_{j a}^{(1)}(p, q)$ to $u_{r s}$ and $u_{p q}$ in (3.10), respectively.

By using the perturbation expansion of the configulations in (3.7) and (2.5), it is easy to obtain asymptotic expansions of measures of inconsistency $\phi(p), \phi_{E}(p)$ and $\phi_{s}(p)$ as follows.

THEOREM 6. Suppose that the multiplicities of the eigenvalues of $\boldsymbol{\Omega}$ are all one. Then, the measures of inconsistency of the configulation $\phi(p), \phi_{E}(p)$ and $\phi_{S}(p)$ can be expanded respectively as

$$
\begin{aligned}
& \phi(p)=\varepsilon^{2} \sum_{\alpha=1}^{p}\left\{\frac{\left(w_{\alpha \alpha}^{(1)}\right)^{2}}{4 \lambda_{\alpha}}+\lambda_{a} \sum_{\beta \neq \alpha} \lambda_{\alpha \beta}^{2}\left(w_{\alpha \beta}^{(1)}\right)^{2}\right\}+O\left(\varepsilon^{3}\right), \\
& \phi_{E}(p)=\varepsilon^{2} \sum_{\alpha=1}^{p}\left\{\frac{\left(w_{\alpha \alpha}^{(1)}\right)^{2}}{4 \lambda_{\alpha}}+\sum_{\beta \neq \alpha} \frac{\left(w_{\alpha \beta}^{(1)}\right)^{2}}{2\left(\lambda_{\alpha}+\lambda_{\beta}\right)}\right\}+O\left(\varepsilon^{3}\right)
\end{aligned}
$$


and

$$
\phi_{S}(p)=\varepsilon^{2}\left[\sum_{a=1}^{p}\left\{\frac{\left(w_{\alpha \alpha}^{(1)}\right)^{2}}{4 \lambda_{\alpha}}+\sum_{\beta \neq \alpha} \frac{\left(w_{\alpha \beta}^{(1)}\right)^{2}}{2\left(\lambda_{\alpha}+\lambda_{\beta}\right)}\right\}-\frac{\left(\sum_{a=1}^{p} w_{\alpha \alpha}^{(1)}\right)^{2}}{4 \sum_{\alpha=1}^{p} \lambda_{\alpha}}\right]+O\left(\varepsilon^{3}\right)
$$

Therefore, the expectations of these measures can be expanded respectively for small $\varepsilon$ as

$$
\begin{aligned}
& E[\phi(p)]=\varepsilon^{2} \sum_{\alpha=1}^{p}\left[\lambda \alpha \sum_{\beta \neq \alpha} \lambda_{\alpha \beta}^{2}\left\{\boldsymbol{\mu}_{(\alpha \beta)}^{\prime} \Theta^{(2)} \boldsymbol{\mu}_{(\alpha \beta)}+\boldsymbol{\mu}_{(\alpha \alpha)}^{\prime} \Theta^{(2)} \boldsymbol{\mu}_{(\beta \beta)}\right\}\right. \\
& \left.+\frac{\mu_{(a \alpha)}^{\prime} \Theta^{(2)} \mu_{(a \alpha)}}{2 \lambda_{a}}\right]+O\left(\varepsilon^{3}\right), \\
& E\left[\phi_{E}(p)\right]=\varepsilon^{2} \sum_{\alpha=1}^{p}\left[\sum_{\beta \neq a} \frac{\left\{\boldsymbol{\mu}_{(\alpha \beta)}^{\prime} \Theta^{(2)} \boldsymbol{\mu}_{(\alpha \beta)}+\boldsymbol{\mu}_{(\alpha \alpha)}^{\prime} \Theta^{(2)} \boldsymbol{\mu}_{(\beta \beta)}\right\}}{\lambda_{\alpha}+\lambda_{\beta}}\right. \\
& \left.+\frac{\boldsymbol{\mu}_{(\alpha a)}^{\prime} \Theta^{(2)} \boldsymbol{\mu}_{(\alpha a)}}{2 \lambda_{\alpha}}\right]+O\left(\varepsilon^{3}\right)
\end{aligned}
$$

and

$$
\begin{aligned}
E\left[\phi_{S}(p)\right]=\varepsilon^{2}[ & \sum_{\alpha=1}^{p}\left\{\sum_{\beta \neq \alpha} \frac{\left\{\boldsymbol{\mu}_{(\alpha \beta)}^{\prime} \Theta^{(2)} \boldsymbol{\mu}_{(\alpha \beta)}+\boldsymbol{\mu}_{(\alpha \alpha)}^{\prime} \boldsymbol{\Theta}^{(2)} \boldsymbol{\mu}_{(\beta \beta)}\right\}}{\lambda_{\alpha}+\lambda_{\beta}}\right. \\
& \left.\left.+\frac{\boldsymbol{\mu}_{(\alpha \alpha)}^{\prime} \Theta^{(2)} \boldsymbol{\mu}_{(\alpha \alpha)}}{2 \lambda_{\alpha}}\right\}-\frac{\sum_{\alpha, \beta=1}^{p} \boldsymbol{\mu}_{(\alpha \beta)}^{\prime} \boldsymbol{\Theta}^{(2)} \boldsymbol{\mu}_{(\alpha \beta)}}{2 \sum_{\alpha=1}^{p} \lambda_{\alpha}}\right]+O\left(\varepsilon^{3}\right) .
\end{aligned}
$$

Also in theorem 6 , again by noting $w_{\alpha \beta}^{(1)}=-(1 / 2) \boldsymbol{\gamma}_{\alpha}^{\prime}\left(2 \boldsymbol{U}^{(\mathbf{l})}\right) \boldsymbol{\gamma}_{\beta}$, we obtain the same expressions of $\phi_{E}(p)$ and $\phi_{S}(p)$ as given in Corollaries 4.2 and 4.4 of Sibson [6], respectively. However, it should be noted that a formula on $E\left[\phi_{s}(p)\right]$ was not provided in Sibson's work. In the next Section, we assess the accuracy of asymptotic expansion formulas derived in this section through two examples. Once we obtain the configulation of the objects by the MDS procedure, it is important to know whether the solution is really structured. One of the methods used to answer this question is to use the asymptotic expansion formulas derived in this section. For an actual calculation, we need to substitute the dissimilarity matrix $\boldsymbol{D}$ to the true $\boldsymbol{\theta}$. This method is illustrated in the following section more concretely.

\section{Numerical examples}

In this section, we give several examples which illustrate our results in the previous section. In the following examples, the propotion of a distance matrix $\boldsymbol{D}$ explained by a $p$ dimensional MDS solution is measured by

$$
\psi_{p}=\left\{\frac{\sum_{\alpha=1}^{p} l_{\alpha}}{\sum_{\alpha=1}^{n} l_{\alpha}}\right\} \times 100
$$

(see, Mardia et al. [3]).

EXAMPLE 1. We consider the following artificial data matrix. 


$$
\boldsymbol{D}_{\boldsymbol{A}}=\left(\begin{array}{ccccc}
0 & & & & \\
1 & 0 & & * & \\
\sqrt{3} & \sqrt{2} & 0 & & \\
2 & \sqrt{2} & 2 & 0 & \\
\sqrt{2} & \sqrt{2} & \sqrt{3} & \sqrt{3} & 0
\end{array}\right) .
$$

The first and the second eigenvalues of YH-transformation of $\boldsymbol{D}_{A}^{(2)}$ are $\lambda_{1}=2.258$ and $\lambda_{2}=1.617$, respectively. Since $\psi_{2}=77.5$ in this case, it is sufficient to represent the objects $R^{2}$. The largest three eigenvalues and true configulation $(\varepsilon=$ 0 ) are listed in Table 1. In Table 2, the performances of the estimated configulations are tabulated for several values of $\varepsilon$. The Values of Mean and Var. (Variance) in the Simula. column are obtained from 1000 replications with $u_{\text {rs }} \sim N(0,1)$. Values of Mean and Var. in the Approx. column are those of (3.5) and (3.6) for eigenvalues and those of (3.8) and (3.9) for configulation, omitting the term of $O\left(\varepsilon^{3}\right)$, respectively. The accuracy of the asymptotic

Table 1.

\begin{tabular}{crrr}
\hline & \multicolumn{3}{c}{$\varepsilon=0$} \\
\cline { 2 - 4 } Eig. Val. & \multicolumn{1}{c}{ First } & Second & Third \\
\cline { 2 - 4 } Config. & \multicolumn{1}{c}{$x_{1}$} & \multicolumn{1}{c}{$x_{2}$} & \multicolumn{1}{c}{$x_{3}$} \\
\hline$O_{1}$ & 0.589 & 0.778 & 0.088 \\
$O_{2}$ & 0.161 & 0.139 & 0.533 \\
$O_{3}$ & 0.719 & -0.927 & -0.137 \\
$O_{4}$ & -1.112 & -0.250 & 0.282 \\
$O_{5}$ & -0.358 & 0.260 & -0.767 \\
\hline
\end{tabular}

Eig. Val.: Eigenvalues,

Config.: Configulation.

Table 2(a).

\begin{tabular}{|c|c|c|c|c|c|c|c|}
\hline \multirow[b]{3}{*}{ Eig. Val. } & \multirow[b]{3}{*}{$\begin{array}{l}\text { Mean } \\
\text { Var. }\end{array}$} & \multicolumn{6}{|c|}{$\varepsilon=0.05$} \\
\hline & & \multicolumn{2}{|c|}{ First } & \multicolumn{2}{|c|}{ Second } & \multicolumn{2}{|c|}{ Third } \\
\hline & & $\begin{array}{c}\text { Simula. } \\
2.271 \\
(0.009)\end{array}$ & $\begin{array}{c}\text { Approx. } \\
2.271 \\
(0.010)\end{array}$ & $\begin{array}{c}\text { Simula. } \\
1.620 \\
(0.008)\end{array}$ & $\begin{array}{c}\text { Approx. } \\
1.618 \\
(0.008) \\
\end{array}$ & $\begin{array}{c}\text { Simula. } \\
0.975 \\
(0.005) \\
\end{array}$ & $\begin{array}{c}\text { Approx. } \\
0.971 \\
(0.005) \\
\end{array}$ \\
\hline Config. & & \multicolumn{2}{|c|}{$x_{1}$} & \multicolumn{2}{|c|}{$x_{2}$} & \multicolumn{2}{|c|}{$x_{3}$} \\
\hline$O_{1}$ & $\begin{array}{l}\text { Mean } \\
\text { Var. }\end{array}$ & $\begin{array}{c}0.587 \\
(0.013)\end{array}$ & $\begin{array}{c}0.588 \\
(0.013)\end{array}$ & $\begin{array}{c}0.766 \\
(0.005)\end{array}$ & $\begin{array}{c}0.766 \\
(0.005)\end{array}$ & $\begin{array}{c}0.083 \\
(0.005)\end{array}$ & $\begin{array}{c}0.084 \\
(0.005)\end{array}$ \\
\hline $\mathrm{O}_{2}$ & $\begin{array}{l}\text { Mean } \\
\text { Var. }\end{array}$ & $\begin{array}{c}0.162 \\
(0.003)\end{array}$ & $\begin{array}{c}0.162 \\
(0.003)\end{array}$ & $\begin{array}{c}0.142 \\
(0.006)\end{array}$ & $\begin{array}{c}0.140 \\
(0.006)\end{array}$ & $\begin{array}{c}0.523 \\
(0.002)\end{array}$ & $\begin{array}{c}0.524 \\
(0.002)\end{array}$ \\
\hline $\mathrm{O}_{3}$ & $\begin{array}{l}\text { Mean } \\
\text { Var. }\end{array}$ & $\begin{array}{c}0.711 \\
(0.018)\end{array}$ & $\begin{array}{c}0.711 \\
(0.016)\end{array}$ & $\begin{array}{c}-0.917 \\
(0.007)\end{array}$ & $\begin{array}{c}-0.918 \\
(0.007)\end{array}$ & $\begin{array}{c}-0.128 \\
(0.007)\end{array}$ & $\begin{array}{c}-0.129 \\
(0.008)\end{array}$ \\
\hline$O_{4}$ & $\begin{array}{c}\text { Mean } \\
\text { Var. }\end{array}$ & $\begin{array}{c}-1.108 \\
(0.002)\end{array}$ & $\begin{array}{c}-1.109 \\
(0.001)\end{array}$ & $\begin{array}{c}-0.240 \\
(0.013)\end{array}$ & $\begin{array}{c}-0.241 \\
(0.012)\end{array}$ & $\begin{array}{c}0.280 \\
(0.002)\end{array}$ & $\begin{array}{c}0.279 \\
(0.002)\end{array}$ \\
\hline$O_{5}$ & $\begin{array}{l}\text { Mean } \\
\text { Var. }\end{array}$ & $\begin{array}{c}-0.352 \\
(0.005)\end{array}$ & $\begin{array}{c}-0.352 \\
(0.004)\end{array}$ & $\begin{array}{c}0.249 \\
(0.016)\end{array}$ & $\begin{array}{c}0.253 \\
(0.016)\end{array}$ & $\begin{array}{c}-0.758 \\
(0.002)\end{array}$ & $\begin{array}{c}-0.759 \\
(0.002)\end{array}$ \\
\hline
\end{tabular}

Simula.: Simulation based on 1000 replications,

Approx.: Values of Asymptotic expansion formulas. 
Table 2(b).

\begin{tabular}{|c|c|c|c|c|c|c|c|}
\hline \multirow[b]{3}{*}{ Eig. Val. } & \multirow[b]{3}{*}{$\begin{array}{l}\text { Mean } \\
\text { Var. }\end{array}$} & \multicolumn{6}{|c|}{$\varepsilon=0.1$} \\
\hline & & \multicolumn{2}{|c|}{ First } & \multicolumn{2}{|c|}{ Second } & \multicolumn{2}{|c|}{ Third } \\
\hline & & $\begin{array}{c}\text { Simula. } \\
2.329 \\
(0.037)\end{array}$ & $\begin{array}{c}\text { Approx. } \\
2.310 \\
(0.043)\end{array}$ & $\begin{array}{c}\text { Simula. } \\
1.620 \\
(0.028)\end{array}$ & $\begin{array}{c}\text { Approx. } \\
1.622 \\
(0.034)\end{array}$ & $\begin{array}{c}\text { Simula. } \\
0.951 \\
(0.022) \\
\end{array}$ & $\begin{array}{c}\text { Approx. } \\
0.942 \\
(0.022) \\
\end{array}$ \\
\hline Config. & & \multicolumn{2}{|c|}{$x_{1}$} & \multicolumn{2}{|c|}{$x_{2}$} & \multicolumn{2}{|c|}{$x_{3}$} \\
\hline$O_{1}$ & $\begin{array}{l}\text { Mean } \\
\text { Var. }\end{array}$ & $\begin{array}{c}0.562 \\
(0.065)\end{array}$ & $\begin{array}{c}0.585 \\
(0.052)\end{array}$ & $\begin{array}{c}0.716 \\
(0.039)\end{array}$ & $\begin{array}{c}0.729 \\
(0.021)\end{array}$ & $\begin{array}{c}0.078 \\
(0.024)\end{array}$ & $\begin{array}{c}0.072 \\
(0.021)\end{array}$ \\
\hline $\mathrm{O}_{2}$ & $\begin{array}{l}\text { Mean } \\
\text { Var. }\end{array}$ & $\begin{array}{c}0.161 \\
(0.013)\end{array}$ & $\begin{array}{c}0.162 \\
(0.013)\end{array}$ & $\begin{array}{c}0.138 \\
(0.025)\end{array}$ & $\begin{array}{c}0.145 \\
(0.025)\end{array}$ & $\begin{array}{c}0.494 \\
(0.013)\end{array}$ & $\begin{array}{c}0.495 \\
(0.011)\end{array}$ \\
\hline $\mathrm{O}_{3}$ & $\begin{array}{l}\text { Mean } \\
\text { Var. }\end{array}$ & $\begin{array}{c}0.693 \\
(0.094)\end{array}$ & $\begin{array}{c}0.686 \\
(0.066)\end{array}$ & $\begin{array}{r}-0.865 \\
(0.034)\end{array}$ & $\begin{array}{r}-0.891 \\
(0.028)\end{array}$ & $\begin{array}{r}-0.114 \\
(0.028)\end{array}$ & $\begin{array}{r}-0.105 \\
(0.032)\end{array}$ \\
\hline$O_{4}$ & $\begin{array}{l}\text { Mean } \\
\text { Var. }\end{array}$ & $\begin{array}{c}-1.079 \\
(0.018)\end{array}$ & $\begin{array}{r}-1.099 \\
(0.007)\end{array}$ & $\begin{array}{c}-0.221 \\
(0.074)\end{array}$ & $\begin{array}{r}-0.216 \\
(0.048)\end{array}$ & $\begin{array}{c}0.268 \\
(0.010)\end{array}$ & $\begin{array}{c}0.270 \\
(0.009)\end{array}$ \\
\hline$O_{5}$ & $\begin{array}{l}\text { Mean } \\
\text { Var. }\end{array}$ & $\begin{array}{c}-0.337 \\
(0.026)\end{array}$ & $\begin{array}{r}-0.335 \\
(0.019)\end{array}$ & $\begin{array}{c}0.231 \\
(0.063)\end{array}$ & $\begin{array}{c}0.232 \\
(0.066)\end{array}$ & $\begin{array}{c}-0.727 \\
(0.008)\end{array}$ & $\begin{array}{r}-0.732 \\
(0.010)\end{array}$ \\
\hline
\end{tabular}

Table 2(c).

\begin{tabular}{|c|c|c|c|c|c|c|c|}
\hline \multirow[b]{3}{*}{ Eig. Val. } & \multirow[b]{3}{*}{$\begin{array}{l}\text { Mean } \\
\text { Var. }\end{array}$} & \multicolumn{6}{|c|}{$\varepsilon=0.2$} \\
\hline & & \multicolumn{2}{|c|}{ First } & \multicolumn{2}{|c|}{ Second } & \multicolumn{2}{|c|}{ Third } \\
\hline & & $\begin{array}{c}\text { Simula. } \\
2.539 \\
(0.127)\end{array}$ & $\begin{array}{c}\text { Approx. } \\
2.465 \\
(0.173)\end{array}$ & $\begin{array}{c}\text { Simula. } \\
1.654 \\
(0.072)\end{array}$ & $\begin{array}{c}\text { Approx } \\
1.639 \\
(0.137)\end{array}$ & $\begin{array}{c}\text { Simula. } \\
0.901 \\
(0.063)\end{array}$ & $\begin{array}{c}\text { Approx. } \\
0.825 \\
(0.089)\end{array}$ \\
\hline \multicolumn{2}{|l|}{ Config. } & \multicolumn{2}{|c|}{$x_{1}$} & \multicolumn{2}{|c|}{$x_{2}$} & \multicolumn{2}{|c|}{$x_{3}$} \\
\hline$O_{1}$ & $\begin{array}{l}\text { Mean } \\
\text { Var. }\end{array}$ & $\begin{array}{c}0.551 \\
(0.194)\end{array}$ & $\begin{array}{c}0.573 \\
(0.209)\end{array}$ & $\begin{array}{c}0.497 \\
(0.174)\end{array}$ & $\begin{array}{c}0.582 \\
(0.085)\end{array}$ & $\begin{array}{c}0.060 \\
(0.088)\end{array}$ & $\begin{array}{c}0.026 \\
(0.084)\end{array}$ \\
\hline $\mathrm{O}_{2}$ & $\begin{array}{l}\text { Mean } \\
\text { Var. }\end{array}$ & $\begin{array}{c}0.155 \\
(0.052)\end{array}$ & $\begin{array}{c}0.165 \\
(0.052)\end{array}$ & $\begin{array}{c}0.122 \\
(0.102)\end{array}$ & $\begin{array}{c}0.165 \\
(0.101)\end{array}$ & $\begin{array}{c}0.380 \\
(0.069)\end{array}$ & $\begin{array}{c}0.380 \\
(0.046)\end{array}$ \\
\hline$O_{3}$ & $\begin{array}{l}\text { Mean } \\
\text { Var. }\end{array}$ & $\begin{array}{c}0.561 \\
(0.385)\end{array}$ & $\begin{array}{c}0.587 \\
(0.267)\end{array}$ & $\begin{array}{r}-0.717 \\
(0.096)\end{array}$ & $\begin{array}{c}-0.780 \\
(0.115)\end{array}$ & $\begin{array}{c}-0.056 \\
(0.097)\end{array}$ & $\begin{array}{c}-0.010 \\
(0.130)\end{array}$ \\
\hline$O_{4}$ & $\begin{array}{l}\text { Mean } \\
\text { Var. }\end{array}$ & $\begin{array}{r}-0.998 \\
(0.075)\end{array}$ & $\begin{array}{c}-1.059 \\
(0.030)\end{array}$ & $\begin{array}{c}-0.075 \\
(0.250)\end{array}$ & $\begin{array}{r}-0.114 \\
(0.192)\end{array}$ & $\begin{array}{c}0.211 \\
(0.041)\end{array}$ & $\begin{array}{c}0.231 \\
(0.037)\end{array}$ \\
\hline$O_{5}$ & $\begin{array}{l}\text { Mean } \\
\text { Var. }\end{array}$ & $\begin{array}{c}-0.269 \\
(0.116)\end{array}$ & $\begin{array}{r}-0.266 \\
(0.079)\end{array}$ & $\begin{array}{c}0.173 \\
(0.199)\end{array}$ & $\begin{array}{c}0.148 \\
(0.266)\end{array}$ & $\begin{array}{c}-0.595 \\
(0.037)\end{array}$ & $\begin{array}{c}-0.627 \\
(0.040)\end{array}$ \\
\hline
\end{tabular}

expansion formulas derived in Section 3 can be observed from the tables.

EXAMPLE 2. The dissimilarity matrix derived from the data introduced in Davison [2] is considered. The original data is concerned with the percentage of workers who were unemployed in five different industries (Private wage/ salary workers, Mining, Manufacturing, Construction and Finance) during five different years. For this data, the dissimilarity matrix $D_{R}$ have been given as

$$
\boldsymbol{D}_{R}=\left(\begin{array}{ccccc}
0 & & & & \\
5.62 & 0 & & * & \\
1.11 & 5.50 & 0 & & \\
12.59 & 13.20 & 12.31 & 0 & \\
5.48 & 7.94 & 5.87 & 18.03 & 0
\end{array}\right)
$$


The first and the second eigenvalues of the YH-transformation of $\boldsymbol{D}_{R}^{(2)}$ are $\lambda_{1}=$ 175.9 and $\lambda_{2}=23.1$, respectively, thus we see that the contribution rate of the configulation to $R^{2}$ is $\psi_{2}=95$. The three largest eigenvalues and the true configulation $(\varepsilon=0)$ are tabulated in Table 3. Similar to the previous example, simulation results and the values of the asymptotic expansion formulas are simultaneously figured in Table 4 . We see that the solution in this example is stable. This is explained from the fact that $\lambda_{1}$ and $\lambda_{2}$ are both very large relative to other eigenvalues.

The mean values of inconsistency measures $\phi(2), \phi_{E}(2)$ and $\phi_{S}(2)$ were obtained from 500 simulations with $u_{r s} \sim N(0,1)$ for several values of $\varepsilon$. The values are listed in the Simula. column of Table 5. Values of the corresponding asymptotic expansion fomulas (3.11), (3.12) and (3.13) in Theorem 6 omitting the term of $O\left(\varepsilon^{3}\right)$ are also figured in the Approx. column. We observe from Table 5 that the present asymptotic expansion formulas in Theorem 6 give good approximate values.

Table 3 .

\begin{tabular}{ccrc}
\hline & \multicolumn{3}{c}{$\varepsilon=0$} \\
\cline { 2 - 4 } Eig. Val. & First & Second & Third \\
\cline { 2 - 4 } Config. & \multicolumn{1}{c}{$x_{1}$} & \multicolumn{1}{c}{$x_{2}$} & \multicolumn{1}{c}{$x_{3}$} \\
\hline$O_{1}$ & -1.540 & 1.326 & 0.246 \\
$O_{2}$ & -1.248 & -4.273 & -0.010 \\
$O_{3}$ & -1.237 & 1.173 & -0.766 \\
$O_{4}$ & 11.02 & 0.586 & 0.173 \\
$O_{5}$ & -6.996 & 1.187 & 0.356 \\
\hline$O_{1}:$ Private wage/salary workers, $O_{2}:$ Mining, \\
$O_{3}$ : Manufacturing, $O_{4}:$ Construction, $O_{5}:$ \\
Finance
\end{tabular}

Table 4(a).

\begin{tabular}{|c|c|c|c|c|c|c|c|}
\hline \multirow[b]{3}{*}{ Eig. Val. } & \multirow[b]{3}{*}{$\begin{array}{l}\text { Mean } \\
\text { Var. }\end{array}$} & \multicolumn{6}{|c|}{$\varepsilon=0.05$} \\
\hline & & \multicolumn{2}{|c|}{ First } & \multicolumn{2}{|c|}{ Second } & \multicolumn{2}{|c|}{ Third } \\
\hline & & $\begin{array}{c}\text { Simula. } \\
175.8 \\
(0.659)\end{array}$ & $\begin{array}{c}\text { Approx. } \\
175.9 \\
(0.662)\end{array}$ & $\begin{array}{c}\text { Simula. } \\
23.15 \\
(0.095)\end{array}$ & $\begin{array}{c}\text { Approx. } \\
23.16 \\
(0.092)\end{array}$ & $\begin{array}{c}\text { Simula. } \\
0.950 \\
(0.137)\end{array}$ & $\begin{array}{c}\text { Approx. } \\
0.903 \\
(0.109)\end{array}$ \\
\hline Config. & & \multicolumn{2}{|c|}{$x_{1}$} & \multicolumn{2}{|c|}{$x_{2}$} & \multicolumn{2}{|c|}{$x_{3}$} \\
\hline$\overline{O_{1}}$ & $\begin{array}{l}\text { Mean } \\
\text { Var. }\end{array}$ & $\begin{array}{c}-1.540 \\
(0.001)\end{array}$ & $\begin{array}{r}-1.540 \\
(0.001)\end{array}$ & $\begin{array}{c}1.328 \\
(0.005)\end{array}$ & $\begin{array}{c}1.325 \\
(0.002)\end{array}$ & $\begin{array}{c}0.179 \\
(0.088)\end{array}$ & $\begin{array}{c}0.201 \\
(0.060)\end{array}$ \\
\hline $\mathrm{O}_{2}$ & $\begin{array}{l}\text { Mean } \\
\text { Var. }\end{array}$ & $\begin{array}{c}-1.248 \\
(0.002)\end{array}$ & $\begin{array}{r}-1.248 \\
(0.001)\end{array}$ & $\begin{array}{c}-4.273 \\
(0.000)\end{array}$ & $\begin{array}{c}-4.273 \\
(0.000)\end{array}$ & $\begin{array}{r}-0.011 \\
(0.000)\end{array}$ & $\begin{array}{r}-0.011 \\
(0.000)\end{array}$ \\
\hline$O_{3}$ & $\begin{array}{l}\text { Mean } \\
\text { Var. }\end{array}$ & $\begin{array}{c}-1.239 \\
(0.001)\end{array}$ & $\begin{array}{c}-1.237 \\
(0.001)\end{array}$ & $\begin{array}{c}1.172 \\
(0.002)\end{array}$ & $\begin{array}{c}1.173 \\
(0.002)\end{array}$ & $\begin{array}{r}-0.734 \\
(0.056)\end{array}$ & $\begin{array}{r}-0.736 \\
(0.020)\end{array}$ \\
\hline $\mathrm{O}_{4}$ & $\begin{array}{l}\text { Mean } \\
\text { Var. }\end{array}$ & $\begin{array}{c}11.02 \\
(0.000)\end{array}$ & $\begin{array}{c}11.02 \\
(0.000)\end{array}$ & $\begin{array}{c}0.586 \\
(0.000)\end{array}$ & $\begin{array}{c}0.586 \\
(0.000)\end{array}$ & $\begin{array}{c}0.183 \\
(0.013)\end{array}$ & $\begin{array}{c}0.177 \\
(0.012)\end{array}$ \\
\hline$O_{5}$ & $\begin{array}{l}\text { Mean } \\
\text { Var. }\end{array}$ & $\begin{array}{r}-6.994 \\
(0.001)\end{array}$ & $\begin{array}{r}-6.996 \\
(0.001)\end{array}$ & $\begin{array}{l}1.186 \\
(0.003)\end{array}$ & $\begin{array}{l}1.187 \\
(0.003)\end{array}$ & $\begin{array}{c}0.383 \\
(0.070)\end{array}$ & $\begin{array}{c}0.368 \\
(0.065)\end{array}$ \\
\hline
\end{tabular}


Table $4(\mathrm{~b})$.

\begin{tabular}{|c|c|c|c|c|c|c|c|}
\hline \multirow[b]{3}{*}{ Eig. Val. } & \multirow[b]{3}{*}{$\begin{array}{l}\text { Mean } \\
\text { Var. }\end{array}$} & \multicolumn{6}{|c|}{$\varepsilon=0.1$} \\
\hline & & \multicolumn{2}{|c|}{ First } & \multicolumn{2}{|c|}{ Second } & \multicolumn{2}{|c|}{ Third } \\
\hline & & $\begin{array}{c}\text { Simula. } \\
175.9 \\
(2.748)\end{array}$ & $\begin{array}{c}\text { Approx. } \\
175.9 \\
(2.651)\end{array}$ & $\begin{array}{c}\text { Simula. } \\
23.18 \\
(0.343)\end{array}$ & $\begin{array}{c}\text { Approx. } \\
23.16 \\
(0.370)\end{array}$ & $\begin{array}{c}\text { Simula. } \\
1.277 \\
(0.727)\end{array}$ & $\begin{array}{c}\text { Approx } \\
0.903 \\
(0.436)\end{array}$ \\
\hline \multicolumn{2}{|l|}{ Config. } & \multicolumn{2}{|c|}{$x_{1}$} & \multicolumn{2}{|c|}{$x_{2}$} & \multicolumn{2}{|c|}{$x_{3}$} \\
\hline$O_{1}$ & $\begin{array}{c}\text { Mean } \\
\text { Var. }\end{array}$ & $\begin{array}{c}-1.537 \\
(0.005)\end{array}$ & $\begin{array}{c}-1.540 \\
(0.005)\end{array}$ & $\begin{array}{c}1.334 \\
(0.009)\end{array}$ & $\begin{array}{c}1.324 \\
(0.024)\end{array}$ & $\begin{array}{c}0.107 \\
(0.211)\end{array}$ & $\begin{array}{r}0.068 \\
(0.541)\end{array}$ \\
\hline $\mathrm{O}_{2}$ & $\begin{array}{l}\text { Mean } \\
\text { Var. }\end{array}$ & $\begin{array}{c}-1.251 \\
(0.007)\end{array}$ & $\begin{array}{c}-1.248 \\
(0.007)\end{array}$ & $\begin{array}{c}-4.272 \\
(0.003)\end{array}$ & $\begin{array}{c}-4.273 \\
(0.007)\end{array}$ & $\begin{array}{c}-0.012 \\
(0.001)\end{array}$ & $\begin{array}{c}-0.011 \\
(0.003)\end{array}$ \\
\hline$O_{3}$ & $\begin{array}{c}\text { Mean } \\
\text { Var. }\end{array}$ & $\begin{array}{r}-1.236 \\
(0.005)\end{array}$ & $\begin{array}{c}-1.237 \\
(0.005)\end{array}$ & $\begin{array}{l}1.172 \\
(0.011)\end{array}$ & $\begin{array}{c}1.172 \\
(0.025)\end{array}$ & $\begin{array}{c}-0.733 \\
(0.199)\end{array}$ & $\begin{array}{r}-0.646 \\
(0.185)\end{array}$ \\
\hline$O_{4}$ & $\begin{array}{l}\text { Mean } \\
\text { Var. }\end{array}$ & $\begin{array}{l}11.02 \\
(0.001)\end{array}$ & $\begin{array}{c}11.02 \\
(0.001)\end{array}$ & $\begin{array}{c}0.582 \\
(0.002)\end{array}$ & $\begin{array}{c}0.586 \\
(0.005)\end{array}$ & $\begin{array}{c}0.203 \\
(0.040)\end{array}$ & $\begin{array}{r}0.186 \\
(0.113)\end{array}$ \\
\hline$O_{5}$ & $\begin{array}{l}\text { Mean } \\
\text { Var. }\end{array}$ & $\begin{array}{c}-6.998 \\
(0.006)\end{array}$ & $\begin{array}{c}-6.995 \\
(0.006)\end{array}$ & $\begin{array}{c}1.183 \\
(0.014)\end{array}$ & $\begin{array}{c}1.188 \\
(0.034)\end{array}$ & $\begin{array}{c}0.434 \\
(0.206)\end{array}$ & $\begin{array}{c}0.402 \\
(0.585)\end{array}$ \\
\hline
\end{tabular}

Table $4(c)$.

\begin{tabular}{|c|c|c|c|c|c|c|c|}
\hline \multirow[b]{3}{*}{ Eig. Val. } & \multirow[b]{3}{*}{$\begin{array}{l}\text { Mean } \\
\text { Var. }\end{array}$} & \multicolumn{6}{|c|}{$\varepsilon=0.2$} \\
\hline & & \multicolumn{2}{|c|}{ First } & \multicolumn{2}{|c|}{ Second } & \multicolumn{2}{|c|}{ Third } \\
\hline & & $\begin{array}{c}\text { Simula. } \\
176.0 \\
(11.00)\end{array}$ & $\begin{array}{c}\text { Approx. } \\
175.9 \\
(10.60)\end{array}$ & $\begin{array}{c}\text { Simula. } \\
23.30 \\
(1.551)\end{array}$ & $\begin{array}{c}\text { Approx. } \\
23.25 \\
(1.481)\end{array}$ & $\begin{array}{c}\text { Simula. } \\
2.318 \\
(3.578)\end{array}$ & $\begin{array}{c}\text { Approx. } \\
2.373 \\
(1.746)\end{array}$ \\
\hline Config. & & \multicolumn{2}{|c|}{$x_{1}$} & \multicolumn{2}{|c|}{$x_{2}$} & \multicolumn{2}{|c|}{$x_{3}$} \\
\hline$O_{1}$ & $\begin{array}{l}\text { Mean } \\
\text { Var. }\end{array}$ & $\begin{array}{c}-1.537 \\
(0.022)\end{array}$ & $\begin{array}{c}-1.541 \\
(0.021)\end{array}$ & $\begin{array}{c}1.322 \\
(0.044)\end{array}$ & $\begin{array}{c}1.320 \\
(0.042)\end{array}$ & $\begin{array}{c}-0.081 \\
(0.475)\end{array}$ & $\begin{array}{c}-0.466 \\
(0.962)\end{array}$ \\
\hline$O_{2}$ & $\begin{array}{l}\text { Mean } \\
\text { Var. }\end{array}$ & $\begin{array}{c}-1.249 \\
(0.028)\end{array}$ & $\begin{array}{c}-1.249 \\
(0.030)\end{array}$ & $\begin{array}{c}-4.270 \\
(0.014)\end{array}$ & $\begin{array}{c}-4.270 \\
(0.013)\end{array}$ & $\begin{array}{c}-0.008 \\
(0.007)\end{array}$ & $\begin{array}{c}-0.011 \\
(0.005)\end{array}$ \\
\hline $\mathrm{O}_{3}$ & $\begin{array}{l}\text { Mean } \\
\text { Var. }\end{array}$ & $\begin{array}{c}-1.239 \\
(0.020)\end{array}$ & $\begin{array}{c}-1.238 \\
(0.020)\end{array}$ & $\begin{array}{c}1.169 \\
(0.047)\end{array}$ & $\begin{array}{c}1.169 \\
(0.046)\end{array}$ & $\begin{array}{c}-0.830 \\
(0.490)\end{array}$ & $\begin{array}{c}-0.288 \\
(0.330)\end{array}$ \\
\hline$O_{4}$ & $\begin{array}{l}\text { Mean } \\
\text { Var. }\end{array}$ & $\begin{array}{c}11.02 \\
(0.007)\end{array}$ & $\begin{array}{c}11.02 \\
(0.007)\end{array}$ & $\begin{array}{c}0.585 \\
(0.010)\end{array}$ & $\begin{array}{c}0.586 \\
(0.010)\end{array}$ & $\begin{array}{c}0.283 \\
(0.098)\end{array}$ & $\begin{array}{c}0.226 \\
(0.202)\end{array}$ \\
\hline$O_{5}$ & $\begin{array}{l}\text { Mean } \\
\text { Var. }\end{array}$ & $\begin{array}{c}-6.997 \\
(0.027)\end{array}$ & $\begin{array}{c}-6.993 \\
(0.026)\end{array}$ & $\begin{array}{c}1.192 \\
(0.062)\end{array}$ & $\begin{array}{c}1.197 \\
(0.060)\end{array}$ & $\begin{array}{c}0.637 \\
(0.532)\end{array}$ & $\begin{array}{c}0.540 \\
(1.041)\end{array}$ \\
\hline
\end{tabular}

Table 5.

\begin{tabular}{|c|c|c|c|c|c|c|}
\hline & \multicolumn{2}{|c|}{$E[\phi(2)]$} & \multicolumn{2}{|c|}{$E\left[\phi_{E}(2)\right]$} & \multicolumn{2}{|c|}{$E\left[\phi_{s}(2)\right]$} \\
\hline & Simula. & Approx. & Simula. & Approx. & Simula. & Approx. \\
\hline$\varepsilon=0.05$ & 0.0167 & 0.0175 & 0.0170 & 0.0174 & 0.0161 & 0.0166 \\
\hline$\varepsilon=0.10$ & 0.0686 & 0.0700 & 0.0621 & 0.0698 & 0.0619 & 0.0664 \\
\hline$\varepsilon=0.15$ & 0.1643 & 0.1575 & 0.1442 & 0.1571 & 0.1355 & 0.1495 \\
\hline$\varepsilon=0.20$ & 0.2760 & 0.2801 & 0.2691 & 0.2793 & 0.2472 & 0.2658 \\
\hline$\varepsilon=0.25$ & 0.4417 & 0.4377 & 0.4026 & 0.4364 & 0.3878 & 0.4153 \\
\hline$\varepsilon=0.30$ & 0.6661 & 0.6303 & 0.5873 & 0.6284 & 0.5362 & 0.5981 \\
\hline$\varepsilon=0.35$ & 0.8754 & 0.8579 & 0.7694 & 0.8553 & 0.7584 & 0.8141 \\
\hline$\varepsilon=0.40$ & 1.1147 & 1.1206 & 0.9629 & 1.1172 & 0.9976 & 1.0633 \\
\hline$\varepsilon=0.45$ & 1. 4362 & 1.4183 & 1.3391 & 1.4139 & 1.1939 & 1.3458 \\
\hline
\end{tabular}

Simula.: Simulation based on 500 replications,

Approx.: Values of Asymptotic expansion formulas. 


\section{Concluding remarks}

In the present paper, various behaviors of the solution of the MDS procedure under a stochastic error model were evaluated for small $\varepsilon$ or large $m$, in which $m$ denotes the number of replications or a measure of confidency of the dissimilarity matrix. Our numerical examples in the previous section were done for some given $\varepsilon=\sigma / m^{1 / 2}$. For an actual use, it is necessary to know the value of $\varepsilon$. Suppose that $m$ dissimilarity matrices $\boldsymbol{D}_{1}, \cdots, \boldsymbol{D}_{m}$ are available and each satisfies $\boldsymbol{D}_{i}=\boldsymbol{\Theta}+\tilde{\boldsymbol{E}}_{i}$, where $\tilde{\boldsymbol{E}}_{i}=\left[\tilde{e}_{i, r s}\right], \tilde{e}_{i, r r}=0, \tilde{e}_{i, r s}=\tilde{e}_{i, s r}$ and $\tilde{e}_{i, r s}$ for $r<s$ and for $i=1, \cdots, m$ are i.i.d. with $N\left(0, \sigma^{2}\right)$. In this case, we can assume the model (2.1) for the sample mean matrix $D$, that is,

$$
\boldsymbol{D}=\frac{1}{m} \sum_{i=1}^{m} \boldsymbol{D}_{i}
$$

which is MLE (Maximum Likelihood Estimate) of $\Theta$. Futher, MLE of $\sigma^{2}$ is

$$
\bar{\sigma}^{2}=\frac{2}{n(n-1) m} \sum_{i=1}^{m} \operatorname{tr}\left(\boldsymbol{D}_{i}-\boldsymbol{D}\right)^{2},
$$

so we obtain an estimate of $\varepsilon$ as $\bar{\varepsilon}=\bar{\sigma} / \mathrm{m}^{1 / 2}$.

Once the solution of MDS for $\boldsymbol{D}$ is obtained, it is important to see whether $\bar{\varepsilon}$ is large or not. If the value of $\hat{\varepsilon}$ is significantly large, then it will be concluded that the analysis based on the MDS solution is not confident.

\section{REFERENCES}

[1] Bhattacharya, R. N. and Ghosh, J. K. (1978). On the validity of the fomal Edgeworth expansion. Ann. Statist., 6, 434-451.

[2] Davison, M. L. (1983). Multidimensional Scaling, John Wiley \& Sons.

[3] Mardia, K. V., Kent, J. T. and Bibby, J. M. (1979). Multivariate Analysis. Academic Press, New York.

[4] Ramsey, J. O. (1977). Maximum likelihood estimate in multidemensional scaling. Psychometrika, 42, 241-266.

[5] Sibson, R. (1978). Studies in the robustness of multidimensional scaling: procrustes statistics. J. R. Statist. Soc., B 40, 234-238.

[6] Sibson, R. (1979). Studies in the robustness of multidimensional scaling: perturbational analysis of classical scaling. J. R. Statist. Soc., B 41, 217-229.

[ 7 ] Siotani, M., Hayakawa, T. and Fujikoshi, Y. (1985). Multivariate Statistical Analysis, American Science Press.

[ 8 ] Torgerson, W. S. (1958). Theory and Methods of Scaling, John Wiley \& Sons. 